\title{
The researches on the analysis of medicinal microbial toxicity spectral curves
}

\author{
Liu Wei-Hong
}

\author{
College of Agriculture and Biology Science,Dali University,Dali,Yunnan 671003,China \\ National natural science foundation of China youth project \# 81102806;
}

Based application fund $2011 \mathrm{fz} 166$ in yunnan province,

Early development state key laboratory of microbial resources opened in 2010

Keywords: magnetic particle; chemiluminescence; medicinal microbial toxicity;

\begin{abstract}
The hidden toxicity in the medicinal microbes is seriously harm to human health and the population are susceptible for the microbes, therefore, the accurate analysis for the medicinal microbial toxicity is essential. This paper presents the analysis of the toxicity spectral curves for the tiny luminescent fungi medicinal microorganisms based on the magnetic particle chemiluminescent methods. By detecting the toxicity of the medicinal microbes with magnetic particles luminescent principles, the paper completes the medicinal microbes toxicity spectral curves analysis via chemistry experiments. The magnetic particles reactions can form stable test objects which can generate stable medicinal microbes toxicity test effects and conclusions. The test method is simple, reliable and it has very good practical application value.
\end{abstract}

\section{Introduction}

With the improvement of medical standards, a variety of different types of medicinal microbes flooded in the domestic pharmaceutical market. Due to the uneven quality of pharmaceutical products, many low-quality medicinal microbes contain harmful toxic ingredients to human beings and they may have a long incubation period which is difficult to be discovered by people. The clinical manifestations of medicinal side effects caused by microbes are complex in recent years. The viral infections due to the abuse of medicinal microbes are increasing greatly with high degree of infection and it seriously threat humans' health. Currently, the main diagnosis methods by using patients' serological to test the toxicity of medicinal microbes in the clinical practices are enzyme-linked immunosorbent assay (ELISA), fluorescent spirochetes absorption test (FTA), medicinal microbial toxicity spirochetes hemagglutination test (TPHA) and so on. However, these methods are very complex with low sensitivity and accuracy ${ }^{[1-3]}$. Therefore, to establish a good method with high sensitivity and accuracy is important for clinical testing of medicinal microbial toxicity.

The magnetic particle chemiluminescent combines the nanotechnology and chemiluminescence technologies, which generates the chemical magnetic enzyme immunoassay method (Magnetic Enzyme Chemiluminescence Immunoassay, MECLIA). The method integrates the immunological methods, magnetic separation technology and chemiluminescent detection technology which can simplify the complex process of antibody cover for simple operation and time saving. The experiments in this paper use human-made magnetic particles as carriers ${ }^{[4-6]}$ and combine the chemiluminescence detection system to establish the magnetic particle chemiluminescent detection methods for detecting the microbial toxicity of medicinal antibodies. The technique has advantages of high sensitivity, high specificity, rapid detection and no radioactive contamination, which can provide timely and accurate test results in real time that can provide basis for medicinal microbial toxicity.

Therefore, under the grim situations of increasing medicinal microbial infection, to accurately detect the medicinal microbial toxicity is the only effective way to deal with medicinal microbes poisoning and diseases. This paper proposes a tiny chemiluminescent fungal medicinal microbes' 
toxicity spectrum curves analysis method based on magnetic particles which applies the light-emitting principles to detect the medicinal microbial toxicity. In the chemical experiments, the magnetic particles reactions are used to form stable detection matters in order to achieve stable chemical detection performance.

\section{Experiments}

\subsection{The reagents and instruments}

The following reagents and instruments are used in the experiments:

$\mathrm{Fe}_{2}\left(\mathrm{SO}_{4}\right)_{3}$ (AR Chengdu Kelong Chemical Reagent Factory), $\mathrm{FeSO}_{4}$ (Chengdu Kelong Chemical Reagent Factory), trisodium citrate (AR Chongqing Oriental Reagent Factory), $\mathrm{HAuCl}_{4}$ (AR Sinopharm Chemical Reagent Co., Ltd.), APTES (AR AIDRICH), 3-mercapto propyl triethoxy silane (Alfa Aesar Co.), aqueous ammonia (30\%), tween-20, tris (hydroxymethyl) aminomethane (Tris base), disodium hydrogen phosphate, dihydrogen phosphate, sodium chloride sodium, high-purity nitrogen, luminol (Lumin01) (Sigma Corporation), right-iodophenol (PIP) (Sigma company).

Visible spectrophotometer UV-2000 (Shanghai younike Instrument Co., Ltd.), incubation oscillator (Shanghai Chi Town Analytical Instruments Manufacturing Co.), UV-visible spectrophotometer (Agilent, USA), incubator (Harbin Dongming medical instrument Factory), microplate reader (U.S. Bio.Tek company), chemiluminescence detector (Germany Berthold Technologies Corporation), vibrating sample magnetometer (U.S. LakeShore VSM-7307).

\subsection{Preparation of $\mathrm{Fe}_{3} \mathrm{O}_{4}$ particles and silane-modified surface}

The experiment uses chemical co-precipitation method to synthesize $\mathrm{Fe}_{3} \mathrm{O}_{4}$ particles which mixes the divalent and trivalent iron salt solution according to the proportions and then alkaline precipitating agent is added to the mixed solution of iron salt. With continuously stirring, the nano-level $\mathrm{Fe}_{3} \mathrm{O}_{4}$ particles can be obtained after a period of reaction.

The total reaction is:

$\mathrm{Fe}^{2+}+2 \mathrm{Fe}^{3+}+8 \mathrm{OH}^{-} \rightarrow \mathrm{Fe} 3 \mathrm{O} 4+4 \mathrm{H} 2 \mathrm{O}$

$\mathrm{n}\left(\mathrm{Fe}^{2+}\right): \mathrm{n}\left(\mathrm{Fe}^{3+}\right): \mathrm{n}\left(\mathrm{OH}^{-}\right)=1: 2: 8($ Molar ratio $)$

The $10 \mathrm{~mL}$ of nano- $\mathrm{Fe}_{3} \mathrm{O}_{4}$ magnetic particles are taken. $90 \mathrm{~mL}$ of mixed solvent with 1:1 proportion of water and ethanol is placed in the $250 \mathrm{~mL}$ round bottom flask. After shaking, $500 \mu \mathrm{L}$ ammonia and $300 \mu \mathrm{L}$ silane reagent are added. At $60^{\circ} \mathrm{C}$ with continuously filling nitrogen, the solvent are stirring for $6 \mathrm{~h}$ with $500 \mathrm{r} / \mathrm{min}$. After the complete reaction, the magnetic particles are separated which are washed repeatedly with water and ethanol until the solution is no longer yellow and there is no more ulfhydryl silylating agent (adding a little 5, 5'-dithiobis (2-nitrophenyl formic acid)). The anhydrous ethanol is used to finalize and the solids contents are measured.

\subsection{Synthesis of the colloidal gold}

The colloidal gold is reduced and precipitated nano-sized gold particles by chloroauric acid $\left(\mathrm{HAuCl}_{4}\right)$ with the effects of the reductant, such as white phosphorus, ascorbic acid, and sodium citrate, which can form negative hydrophobic gum solution. According to the synthesis method reported in Frens, the trisodium aqueous solution with smaller scale of nano-gold particles can be synthesized. The $200 \mathrm{~mL}$ of $0.01 \%$ aqueous solution is filled in the four-necked flask to shake at $500 \mathrm{r} / \mathrm{min}$ and pre-heated in 5 minutes. The appropriate amount of $1 \%$ sodium citrate solution is added until the solution color doesn't change any more. The solution is cooled to room temperature and reflow for $15 \mathrm{~min}$. The colloidal gold particles with size of $15 \sim 20 \mathrm{~nm}$ can be obtained and they are orange-red which are placed in a brown bottle and stored at $4{ }^{\circ} \mathrm{C}$.

\subsection{Preparation of TP mixed antigen magnetic particles}

The $0.4 \mathrm{~mL}$ of golden magnetic particles in the centrifuge tube are magnetically separated. The $0.5 \mathrm{~mL}$ of coupling buffer solution (20 mmol / L, pH7.4) is used for wash twice in order to balance $\mathrm{pH}$. The magnetic particles are separation and the supernatant is discarded. The $0.05 \sim 0.5 \mu \mathrm{g} / \mu \mathrm{L}$ antigen solution is added in the magnetic particles and placed in the shaker by 200rpm and react for $30 \mathrm{~min}$ at $37^{\circ} \mathrm{C}$. The magnetic is separated and the coated particles are repeatedly washed with 
coupling buffer solution with antigen. Some blocking solution is added into the incubation oscillator and reacted (200rpm / min, $37{ }^{\circ} \mathrm{C}, 1 \mathrm{~h}$ ) in order to isolate the magnetic particles. PBST is used to wash for 3 times and finally the particles are stored in $1 \mathrm{~mL}$ of PBS and they are mixed evenly for future use.

\subsection{Formation of the TP magnetic particle chemiluminescent detection}

$30 \mu \mathrm{L}$ TP antigen-coated magnetic particles, $50 \mathrm{pL}$ negative/positive serum and HRP labeled antigen are added into the reaction tube. They are placed in the constant temperature oscillator and shake in $30 \mathrm{~min}$ at $37^{\circ} \mathrm{C}$. The reaction tubes are took out and the magnetic is separated. The supernatant is discarded. $500 \mu \mathrm{L}$ of PBST is used to repeatedly wash for six times. The magnetic is separated again and the supernatant is discarded. $10 \mu \mathrm{L}$ of PBS is added. The samples in each tube are moved to the corresponding specific chemiluminescence 96-hole plate. $20 \mu \mathrm{L}$ of $0.05 \%$ Tween-20, Luminol, H2O2, and PIP are added to the samples in the 96-hole plate. The prepared 96-hole plate is placed in the chemiluminescence detector.

\section{Experimental results and analysis}

\subsection{Preparation and characterization of the magnetic particles}

The magnetic particle synthesis requires two steps: (1) the magnetic $\mathrm{Fe}_{3} \mathrm{O}_{4}$ particles and colloidal gold particles should be synthesized respectively and the $\mathrm{Fe}_{3} \mathrm{O}_{4}$ particles should be processed with silane reagent to make the surface join - $\mathrm{SH}$ group; (2) Fe3O4 particles should be mixed and react with colloidal gold to obtain the gold magnetic particles with the average particle size of $2 \sim 3 \mu \mathrm{m}$.

Due to the specific absorption peak of $520 \mathrm{~nm}$ in the visible region for the colloidal gold particles, the experiments add $15 \sim 20 \mathrm{~nm}$ colloidal gold particles to the magnetic particles processed by sulfhydryl for complete reaction. The UV spectrophotometer is used to real-time detect the optical absorption of the colloidal gold and the synthesized magnetic particles. The detection results are shown in Fig. 1 . The results illustrate the longer the reaction time, the smaller of the absorption value at $500 \mathrm{~nm}$ in the peak figure and the smaller of the colloidal gold particles are. The gold nano-particles are absorbed in the surface of the functional magnetic particles. The dynamic monitor of the $400 \mathrm{~nm}-800 \mathrm{~nm}$ visible spectrums in the gold magnetic particles formation is shown in Fig. 1.

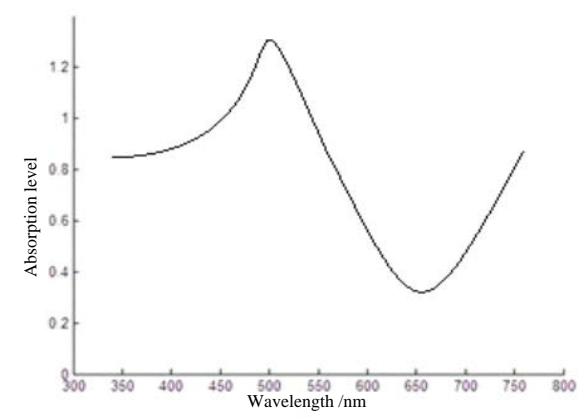

Fig. 1 The dynamic monitor of 400nm-800nm visible lights absorption spectrums in gold magnetic particles formation process

From the absorption spectrum in Fig. 1, in the different band and the different wavelength, the absorption levels of each sample are different. The curve analysis results of the medicinal microbes' toxicity spectrums can be obtained based on the evaluation of the absorption levels.

The vibrating sample magnetometer is used to measure the hysteresis loops of the synthesized gold particles and $\mathrm{Fe}_{3} \mathrm{O}_{4}$ respectively. From the results in Fig. 2, the gold magnetic particles have superparamagnetic. The saturation magnetization of the magnetic particles is $38 \mathrm{emu} / \mathrm{g}$. Therefore, the magnetic particles may be repeatedly washed and separated in the external magnetic fields. The hysteresis loop of the synthesized magnetic particles is as shown in Fig. 2. 


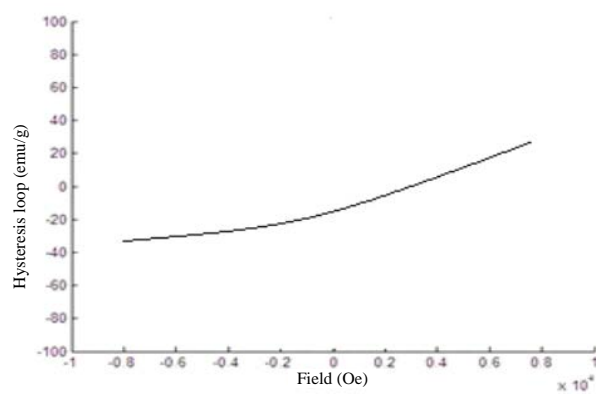

Fig. 2 The synthesized magnetic particles hysteresis loop

\subsection{The immobilization of the magnetic particle surface antibody and the analysis of the luminescent system performance}

Since the surface of the magnetic particles contain a large amount of colloidal gold particles, the protein molecules can easily attain the antigen on the surface of the gold magnetic particles with the electrostatic interaction, hydrophobic interactions, and sulfhydryl function of the side chain of a cysteine residue. The prior to coated TP antigen solution and the after-coated remaining solution is detected with UV absorption which illustrates the TP antigen solution is coated on the surface of the gold magnetic particles.

The separated samples of the positive medicinal microbes are diluted by 10 times, 100 times, 150 times, 250 times, 500 times and 1000 times according to the previous test procedure in order to the detect the relative fluorescence strength of each positive medicinal microbes separate dilutions. As shown in the Fig. 3, the positive samples after diluting by 10 1000 times have better linear property and the correlation coefficient is more than 0.999 .

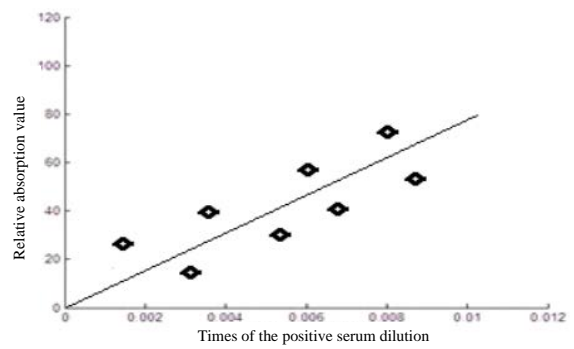

Fig. 3 The standard curve of the magnetic particle chemiluminescent microbiological test for medicinal patient TP antibody

\section{Conclusions}

This paper focuses on the toxicity spectral curves analysis issues of the medicinal microbes, which analyzes a method for the medicinal microbes toxicity spectral curves based on the magnetic particle chemiluminescent methods. By detecting the existence and variants of the medicinal microbes toxicity with luminescent principles, the stable detection objects can be formed with magnetic particles reactions to provide with stable chemistry detection performance. The test method is simple, reliable and it has very good practical application value.

\section{References}

[1] Qian Jun Li Jiuming, only is jin fang, tain things. Of the construction of the whole cell microbial sensor based on e. coli and its application in the acute biological toxicity test [J]. Journal of analytical chemistry: 2013.5:738-743

[2] Justin quek, Sun Zhi, Muscovite, mica muscovitum, van. Cosmetics toxicity test method of new progress

[J]. Chinese journal of frontier health and quarantine, 2012, 35 (6) : 419-424.

[3] Gao Jingxian, tianjin office, golden dream, YanXiaoLi. Medical ultrasonic coupling agent in vitro cytotoxicity test study [J]. Chinese journal of medical apparatus and instruments. 2013.3:210-212

[4] Cui Yali, Wang Yani, Hui Wenli, et al., The short of GoldMag nano - particles and its application for antibody immobilization. The Biomedical Microdevices, 2005, 7 (2) : 153-156.

[5] Kinoshita T, Seino S, Maruyama H, et al. The Influence of the size distribution on the magnetocaloric effect of the super paramagnetic nanocomposite of Fe3O4 / Au. J Alloys \& Comp, 2004, 365:281-285.

[6] jia-qiang pan, xue-wen hou. OsRUS2.1 yeast two hybrid prey carrier construction and its effect on the cytotoxicity and the activation detection [J]. Journal of guangxi plants. 2013.1:76-81 